\title{
Contraception in the cosmos: the combined oral contraceptive pill in space
}

\section{Ali Murad}

\section{Introduction}

On 12 April 1961, Yuri Gagarin became the first human to travel into space. His 2-hour voyage aboard the Vostok 3KA-2 definitively demonstrated that humans could survive in space. In the 55 years since Gagarin's historic flight, hundreds of astronauts and cosmonauts have travelled into space, mankind has visited the moon and it is now realistic to believe that a manned mission to Mars will commence in the foreseeable future.

Space is a uniquely harsh environment. The lack of oxygen and presence of microgravity and space radiation predispose astronauts to a wide range of health problems. The microgravity, busy mission schedules and lack of medical equipment make treating acute health problems during spaceflight extremely difficult. Prevention of disease is therefore of the utmost importance and by ensuring astronauts remain healthy, aerospace medicine physicians make a vital contribution to the success of missions into space.

The combined oral contraceptive pill (COC) is one medication that could be used to prevent health problems in space. Whilst there is no official requirement for them to do so, the vast majority of the National Aeronautics and Space Administration's (NASA) female astronauts choose to use the COC whilst in space (P Stepaniak, NASA Flight Surgeon, personal communication, 23 October 2006). Whilst much is known about the physiological effects of the COC on Earth, there has been no discussion of the potential benefits and disadvantages of its use in space. Such a discussion must take into account the effects of spaceflight on human physiology and the fact that modern female astronauts are a highly selected, motivated and, in general, healthy group of individuals.

This article aims to review the potential benefits and disadvantages of using the COC during long-duration stays in space. It begins with a discussion of the gynaecological effects of the COC. It then examines how the COC might influence the effects of spaceflight on the human musculoskeletal system. Finally, it explores ways in which the COC could affect other systems of the body in ways that are relevant to spaceflight.

\section{Gynaecological effects of the COC in space Contraceptive effects}

Current astronaut crews typically contain both men and women. This is advantageous because it allows astronaut selection to take place from a wide potential pool of candidates and also because mixed-gender crews are more likely to enjoy a favourable social climate than single-sex crews. This has been demonstrated by a study in a French polar station ${ }^{1}$ and by a European Space Agency Study, which studied the behaviour of groups isolated in a hyperbaric chamber. ${ }^{2}$

J Fam Plann Reprod Health Care 2008; 34(1): 55-59

(Accepted 24 September 2007)

North Bristol NHS Trust, Frenchay Hospital, Bristol, UK Ali Murad, BA, BMBCh, Medical Student

Correspondence to: Dr Ali Murad, North Bristol NHS Trust, Room 5, Frenchay Doctors' Mess, Frenchay Hospital, Bristol BS16 1LE, UK. E-mail: Alimurad@doctors.org.uk
However, mixed-gender crews raise the possibility that heterosexual sexual intercourse could occur in space. The average age of a new female astronaut is 32 years $^{3}$ and so such intercourse could result in conception. The ability of vertebrates to mate and conceive offspring whilst in space has already been demonstrated by an historic experiment carried out onboard the International Microgravity Laboratory in which a pair of Medaka fish mated, conceived and hatched eggs. 4

Such a pregnancy must be avoided for the sake of the fetus and the mother. In vitro cultures of human stem cells in space have been shown to grow less rapidly than control cultures grown on Earth. ${ }^{5}$ This suggests that microgravity, or some other characteristic of space, might predispose a fetus to developmental abnormality. In addition, the high levels of galactic cosmic radiation to which astronauts are exposed, even when within their spacecraft, ${ }^{6,7}$ could be teratogenic. Furthermore, pregnancy is associated with significant maternal morbidity and mortality 8,9 and should a pregnancy-induced illness develop during spaceflight, available treatment modalities would be limited. For example, if a crewmember were to develop an ectopic pregnancy that was not amenable to medical treatment with methotrexate, ${ }^{10,11}$ no surgical treatment could be offered.

The COC could be used in space to prevent pregnancy from occurring. It is a highly effective contraceptive and when used correctly it has a Pearl index (number of pregnancies per 100 years of person use) of approximately 0.1.12,13 Whilst actual first-year failure rates are approximately $8 \%, 13,14$ astronauts are highly motivated individuals who could be expected to use the COC correctly. The COC therefore has the potential to prevent pregnancies in space, which could prove highly beneficial.

\section{Menstrual problems}

Menstrual problems could also be deleterious to spaceflight. More than $50 \%$ of women suffer from menstrual pain (dysmenorrhoea). ${ }^{15,16}$ In 1 in 20 women, this pain is sufficiently severe to interfere with daily activities. ${ }^{17}$ Excessively heavy menstrual bleeding (menorrhagia) is another common menstrual complaint that can interfere with function and cause iron deficiency anaemia.

Menstruation in space may result in dysmenorrhoea, menorrhagia and anaemia. This may be of particular importance because spaceflight is associated with a reduction in haemoglobin concentration, 18 further predisposing astronauts to developing anaemia. In addition, some authorities believe that menstruation in microgravity predisposes female astronauts to endometriosis by enhancing 'retrograde menstruation', 19 the process by which viable endometrial cells are carried backwards through the Fallopian tubes during menstruation. Female astronauts may already be predisposed to endometriosis due to the high levels of radiation encountered in space. Studies using irradiated monkeys have identified radiation as a predisposing factor for the development of endometriosis ${ }^{20-23}$ and in the 1970s NASA found this evidence sufficiently compelling to prohibit females with any history of endometriosis from becoming astronauts. 18 Dysmenorrhoea, menorrhagia or endometriosis in a female astronaut in space could prevent her from fulfilling her duties $^{6}$ and potentially jeopardise the success of a mission. 
The COC is an effective and safe treatment for dysmenorrhoea, ${ }^{15}$ menorrhagia ${ }^{24}$ and endometriosis. ${ }^{25}$

\section{Malignant gynaecological conditions}

Malignant gynaecological conditions are also of importance. Endometrial cancer is a common malignancy and $25 \%$ of tumours occur in premenopausal women. ${ }^{26}$ Of relevance, nulliparity is associated with a three-fold increased risk of developing the disease and $70 \%$ of female astronauts have not had a child by the time they enter space. ${ }^{18}$ Ovarian cancer is the most common gynaecological cause of death. ${ }^{26}$ It is more commonly found in women over the age of 40 years and is also more common in women who have not had children. Female astronauts are therefore also at risk of developing ovarian cancer.

Presentation of such cancers during a long-duration space mission (e.g. a mission to Mars) could prove disastrous. It is estimated that such a mission would take at least 3 years and no early return to Earth for treatment would be possible.

Several large-scale trials have demonstrated that the $\mathrm{COC}$ reduces the risk of developing endometrial cancer or ovarian cancer by up to $50 \% .27,28$ Thus, in combination with careful pre-flight screening, the COC could be a useful method of minimising the risk of gynaecological malignancies during a mission of long duration.

\section{Musculoskeletal effects of the COC in space \\ Bone loss}

Spaceflight is associated with an increase in bone reabsorption and a decrease in bone formation. Bone loss in space is thought to arise due to a combination of reduced skeletal loading, low light levels and high ambient $\mathrm{CO}_{2}$ concentrations. ${ }^{6}$

On Earth, gravity plays a vital role in the maintenance of bone strength. This is demonstrated by studies on patients who are wheelchair-bound. Although such patients lose considerable bone mass in their lower extremities, their lumbar spine, which still experiences gravitational loading, remains unaffected. ${ }^{29}$ In space, gravitational mechanical loading of bone does not occur and bone remodelling is reduced. This adversely affects the integrity of the bone.

Activated vitamin D is an important factor in skeletal mineralisation. One step in the formation of active vitamin $\mathrm{D}$ is the isomerisation of pro-vitamin D3 into active vitamin D330 through the action of ultraviolet light on the skin. During long stays in space, astronauts suffer a paucity of ultraviolet light exposure, resulting in poor mineralisation of bone. ${ }^{6}$

The third threat to an astronaut's skeleton comes from the high $\mathrm{CO}_{2}$ concentrations onboard modern spacecraft. Chronic exposure to $\mathrm{CO}_{2}$ results in a respiratory acidosis . To maintain homeostasis, bone reabsorption increases, providing carbonates and phosphates to act as a buffer. 31,32 On Earth, $\mathrm{CO}_{2}$ comprises $0.03 \%$ of the atmosphere. However, in a space shuttle it can make up $1 \%$ of the atmosphere! 6

Studies from the Skylab programme in the 1970s showed that this bone loss in astronauts can be dramatic. A 1 -month stay in space resulted in a loss of $0.3 \%$ of total body calcium. ${ }^{33}$ More recent studies have shown that daily urinary calcium loss increases with increasing duration in space, suggesting that bone loss accelerates the longer an astronaut stays in space (Figure 1). Data from the

\section{Mean Urinary Calcium in Space}

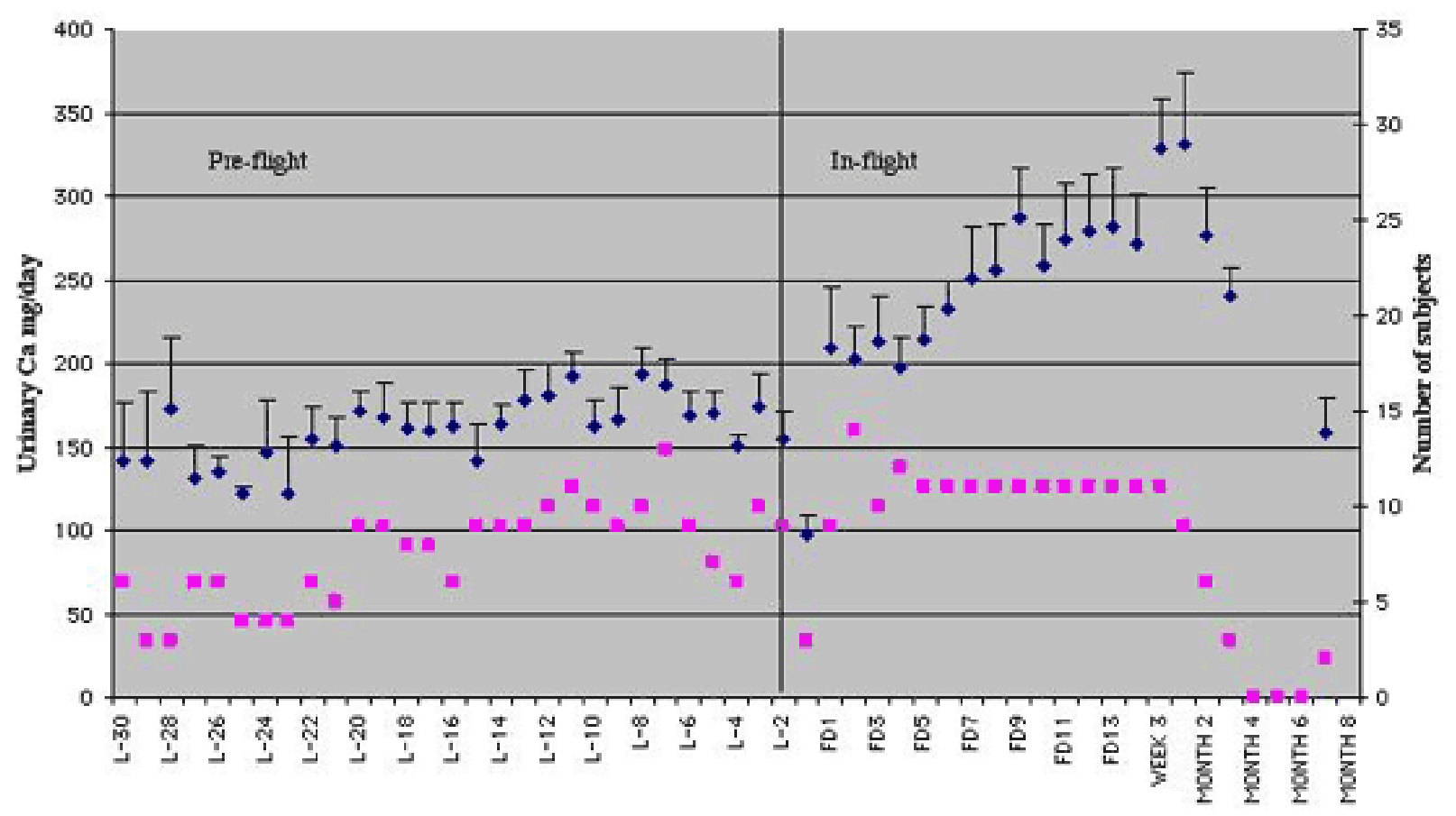

1. Compiled from Data in the Life Sciences Data Archive.

2. Data from mission Gemini VII, Skylab 2-4, Shuttle, Salyut 7 ,

$\downarrow$ Mean Urinary Caloium number of sub jeots

Soyuz 9.

3. Life Sciences Data Archive does not independently verify results.

4. L- means launch minus $\mathrm{x}$-days, FD means flight day.

Figure 1 Daily urinary calcium excretion increases with increasing duration in space ${ }^{34}$ 


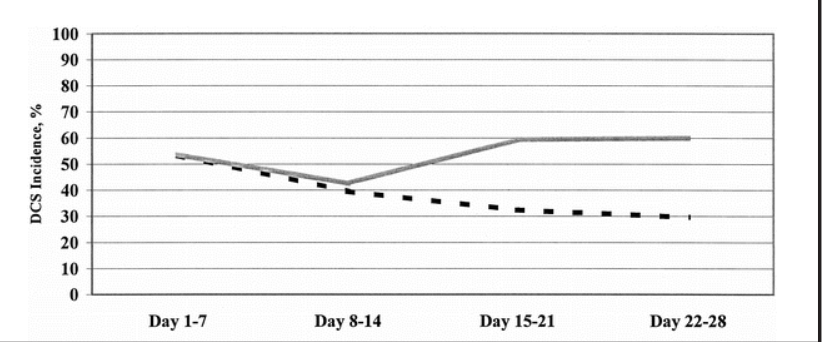

Figure 2 Decompression sickness (DCS) susceptibility in the second half of the menstrual cycle is increased by the combined oral contraceptive pill (COC). 43 The dotted line represents COC use and the solid line represents no COC use

International Space Station has demonstrated that weightbearing areas of the skeleton, such as the hip, may lose up to $1.7 \%$ of bone mineral each month. ${ }^{34}$ Astronauts on a long-duration spaceflight are therefore at a greatly increased risk of fracture. ${ }^{6}$

The current NASA space programme uses exercise regimes, involving high-impact loading of the upper extremities, to minimise bone loss. Whilst these regimes have some effect, an astronaut would need to exercise for more than 7 hours each day to entirely prevent bone loss. ${ }^{6}$ This is impractical and as a consequence some bone loss does occur.

The COC has been shown to increase bone density in premenopausal women 35,36 and the magnitude of this effect may be comparable to that of bisphosphonates. ${ }^{37}$ The bone-sparing effect is greatest in women who have been taking the COC for 3 years or more.

Administration of the COC during prolonged spaceflight could therefore slow the rate of bone reabsorption and this effect could be maximised by ensuring that female astronauts begin taking the COC before they travel into space.

\section{Decompression sickness}

Astronauts' joints are also at risk in space. Decompression sickness (DCS) occurs when a reduction in atmospheric pressure causes nitrogen dissolved in blood and tissues to exit from a solution and form bubbles. ${ }^{6}$ If these bubbles expand in or around joints they cause pain (Type 1 DCS). If the bubbles expand within the central nervous system, they can cause stroke or paralysis (Type 2 DCS). Astronauts are at risk of DCS whilst performing spacewalks [or extravehicular activity (EVA)] because of the pressure difference between their spacecraft and the EVA suit. The difference is large and on Earth would be equivalent to immediately ascending from sea level to

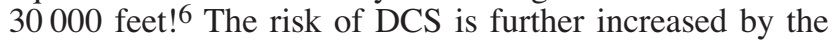
fact that strenuous activity, such as that performed by an astronaut on an EVA, makes DCS more likely. ${ }^{38}$

Experimental studies predict that large numbers of astronauts will develop clinical DCS in space. Waligora et al. exposed 38 subjects to a simulated altitude of 30000 feet after having them complete a 6-hour oxygen prebreathe similar to that used by astronauts and found that $11 \%$ of these subjects developed clinical symptoms of DCS. 39

It is therefore surprising that there has only been one recorded incident of DCS amongst NASA astronauts (P Stepaniak, NASA Flight Surgeon, personal communication, 23 October 2006). There are several theories as to why the incidence of DCS in space is so low, but the most plausible one is that DCS does occur frequently but is underreported. Astronauts are aware that reporting an episode of DCS may jeopardise their participation in future missions and there is therefore a strong disincentive to report symptoms (S Helms, retired NASA astronaut, personal communication, 26 October 2006). Regardless, DCS could cause morbidity in astronauts and efforts should be made to minimise its occurrence.

There is emerging evidence that the occurrence of DCS in women is related to the menstrual cycle. Several studies of decompression sickness in divers or using altitude chambers have found that DCS occurs more frequently in women who are menstruating or who are approaching menstruation. 40,41 The exact reasons for this are not known but it has been suggested that it could be due to tissue fluid shifts affecting gas uptake and elimination. ${ }^{42}$

Two recent studies have also concluded that taking the COC may predispose women to DCS. The first study exposed 100 women subjects to low pressure atmospheres in an altitude chamber. ${ }^{43}$ The study found that in the second half of the menstrual cycle, users of the COC were twice as likely to suffer from DCS symptoms as non-users (Figure 2). The second study reported on retrospective questionnaires completed by 150 women who had suffered from DCS. ${ }^{44}$ This study found a significantly increased incidence of DCS in women over the age of 33 years who used the COC. Since many female astronauts will be over the age of 33 years by the time they enter space, such a finding could be significant.

The evidence linking the COC to DCS remains weak and the importance of any effect is uncertain. However, this situation may change as more research is performed.

\section{Other effects of the COC relevant to spaceflight \\ Breast cancer}

Breast cancer is the most common female malignancy and affects $13 \%$ of women. Whilst breast cancer is more common in the elderly, women of all ages can develop the disease. Since nulliparity is a risk factor for the development of breast cancer, 45 female astronauts may be at a higher risk of developing the disease than other women of their age.

If a female astronaut was to develop breast cancer during a 3-year mission to Mars the consequences could be disastrous. Returning to Earth for therapy would be impossible and by the time definitive treatment was available the individual's prognosis may be poor.

The COC may increase the risk of breast cancer in users. One meta-analysis of 150000 women concluded that usage of the COC was associated with a relative risk of developing breast cancer of 1.24.46

If the COC does predispose to breast cancer, every effort must be made to prevent it from occurring during a long-duration mission. Some protection could be provided by exhaustive pre-screening before flight and excluding candidates who are thought to have a significant risk of developing breast cancer.

\section{Motion sickness}

Motion sickness is a collection of symptoms (nausea, vomiting, drowsiness, cold sweats) that occur in novel motion environments. Its aetiology is incompletely understood, but more than $80 \%$ of astronauts develop motion sickness symptoms after entering space. Severe, uncontrollable motion sickness could prevent an astronaut from fulfilling their duties and must therefore be avoided.

Two recent, small studies have suggested that susceptibility to motion sickness is related to the menstrual cycle. The first study examined 27 women participants in the Global Challenge Round the World Yacht Race. ${ }^{47}$ Participants completed the Reason Motion Sickness 


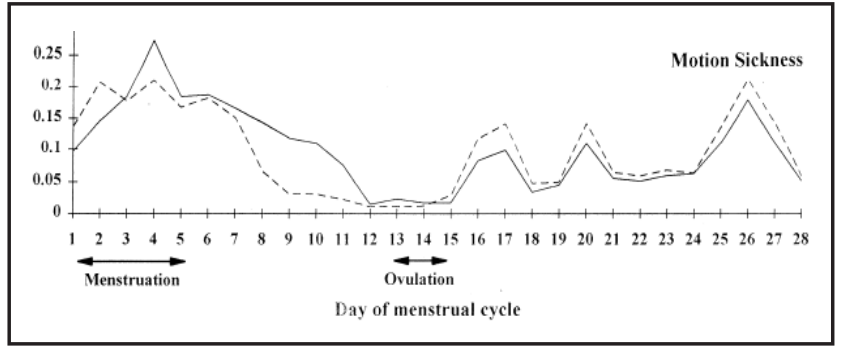

Figure 3 Motion sickness susceptibility varies with the menstrual cycle. The dotted line represents participants suffering from motion sickness and migraine and the solid line represents participants suffering from motion sickness alone 53

Questionnaire, ${ }^{48}$ kept logs of motion sickness symptoms and provided detailed information about their menstrual cycle. From analysing this information, the authors concluded that "motion sickness was related to the menstrual cycle" and that women were most at risk from Day 0 to Day 5 of their cycle. The effect was large and incidence at Day 5 of the cycle was five-fold greater than incidence at Day 15 (Figure 3). ${ }^{47}$ The more recent study used a cross-coupled mechanical chair to induce motion sickness in the laboratory environment amongst 12 women volunteers, ${ }^{49}$ concluding that women were most susceptible to motion sickness at Day 5 of their menstrual cycle and that susceptibility to motion sickness is reduced in the latter half of the cycle.

Unfortunately there is as yet no satisfactory explanation as to why this should be the case.50,51 Regardless, if changes in endogenous hormone levels affect motion sickness susceptibility, exogenous hormone administration should have similar effects. At the time of writing, this issue remains un-addressed by the scientific literature. 52

\section{Venous thromboembolism}

Venous thromboembolism (VTE) is a serious cause of morbidity and mortality. Unfortunately there is very little research into how spaceflight affects the risk of thrombus formation. There are three reasons why astronauts may be at an increased risk of VTE compared to the general population. First, before takeoff and before landing astronauts spend several hours stationary. Studies on airplane passengers have shown that immobilisation, perhaps even for as little as 4 hours, ${ }^{54}$ is a strong risk factor for thrombus formation. In addition, many astronauts intentionally dehydrate themselves in order to avoid needing to urinate in the periods before takeoff and landing (D Cohen, research physiologist at the Kennedy Space Center, personal communication, 21 October 2006). Dehydration also increases the risk of thrombus formation. ${ }^{55}$ The third reason that astronauts may be at an increased risk of thrombus formation is that spaceflight is associated with a reduction in circulating plasma volume. The exact mechanism for this remains unclear, but the effect can be significant and has been measured as a $17 \%$ reduction after only 24 hours in space! 6 This may have the effect of concentrating components involved in thrombus formation, thereby increasing the likelihood of thrombus occurring.

If a venous thrombus or venous embolus was to develop during spaceflight, medicinal treatments, such as the use of thrombolytic and anticoagulant drugs, could be administered in space. However, more drastic treatments, such as surgical clot removal or the insertion of a vena cava filter, would not be possible.

Use of the COC is known to increase the risk of venous thrombosis $^{56}$ and the risk is particularly great in obese women and women aged over 40 years. ${ }^{57}$
Whilst fitness requirements make obesity in astronauts unlikely, care must still be taken to avoid the development of VTE in female astronauts taking the COC, particularly because the majority of experienced astronauts are aged over 40 years.

\section{Conclusions}

The COC has been extensively studied and is known to have a wide range of physiological effects. Some of these effects are particularly relevant to the unique environment of space.

The COC could benefit female astronauts through its contraceptive action, by preventing menstrual problems, by reducing the risk of gynaecological cancers and by preventing bone loss in space. These effects are well established and would be of benefit during a long-duration space mission. Such benefits must be balanced against the potential risks: the possible increased risk of breast cancer and VTE.

There are also early indications that the COC could increase susceptibility to both DCS and motion sickness. It is important that as more research is completed, the suitability of using the COC in space is kept under constant review.

\section{Statements on funding and competing interests}

Funding None identified.

Competing interests None identified.

\section{References}

1 Rosnet E, Jurion S, Cazes G, Bachelard C. Mixed-gender groups: coping strategies and factors of psychological adaptation in a polar environment. Aviat Space Environ Med 2004; 75(7 Suppl.): C10-C13.

2 Manzey D, Kanas N. Space Psychology and Psychiatry. Dordrecht, The Netherlands: Kluwer Academic Publishers, 2003.

3 Jones JA, Jennings R, Pietryzk R, Ciftcioglu N, Stepaniak P. Genitourinary issues during spaceflight: a review. Int $\mathrm{J}$ Impot Res 2005; 17(Suppl. 1): S64-S67.

4 ljiri K. Fish mating experiment in space - what it aimed at and how it was prepared. Biol Sci Space 1995; 9: 3-16.

5 Davis TA, Wiesmann W, Kidwell W, Cannon T, Kerns L, Serke $C$, et al. Effect of spaceflight on human stem cell hematopoiesis: suppression of erythropoiesis and myelopoiesis. J Leukoc Biol 1996; 60: 69-76.

6 Buckley J. Space Physiology. Oxford, UK: Oxford University Press, 2006

7 Fry RJ. Radiation effects in space. Adv Space Res 1986; 6: 261-268.

8 Khan KS, Wojdyla D, Say L, Gülmezoglu AM, Van Look PF. WHO analysis of causes of maternal death: a systematic review. Lancet 2006; 367: 1066-1074.

9 Ronsmans C, Graham WJ. Maternal mortality: who, when, where, and why. Lancet 2006; 368: 1189-1200.

10 Barnhart KT, Gosman G, Ashby R, Sammel M. The medical management of ectopic pregnancy: a meta-analysis comparing "single dose" and "multidose" regimens. Obstet Gynecol 2003; 101: 778-784.

11 Shima TL. Ectopic pregnancy. Top Emerg Med 2002; 24: 12-20.

12 Audet MC, Moreau M, Koltun WD, Waldbaum AS, Shangold G, Fisher AC, et al.; ORTHO EVRA/EVRA 004 Study Group. Evaluation of contraceptive efficacy and cycle control of a transdermal contraceptive patch vs an oral contraceptive: a randomized controlled trial. JAMA 2001; 285: 2347-2354.

13 Fu H, Darroch JE, Haas T, Ranjit N. Contraceptive failure rates: new estimates from the 1995 National Survey of Family Growth. Fam Plann Perspect 1999; 31: 56-63.

14 Trussell J, Vaughan B. Contraceptive failure, method-related discontinuation and resumption of use: results from the 1995 National Survey of Family Growth. Fam Plann Perspect 1999; 31: 64-72, 93.

15 Proctor ML, Roberts H, Farquhar CM. Combined oral contraceptive pill (OCP) as treatment for primary dysmenorrhoea. Cochrane Database Syst Rev 2001; (4): CD002120. 
16 Patel V, Tanksale V, Sahasrabhojanee M, Gupte S, Nevrekar P. The burden and determinants of dysmenorrhoea: a populationbased survey of 2262 women in Goa, India. Br J Obstet Gynaecol 2006; 113: 453-463.

17 Latthe P, Latthe M, Say L, Gulmezoglu M, Khan KS. WHO systematic review of prevalence of chronic pelvic pain: a neglected reproductive health morbidity. BMC Public Health 2006; 6: 177.

18 Jennings RT, Baker ES. Gynecological and reproductive issues for women in space: a review. Obstet Gynecol Surv 2000; 55: 109-116.

19 Sampson JA. Peritoneal endometriosis due to the menstrual dissemination of endometrial tissue into the peritoneal cavity. Am J Obstet Gynecol 1927; 14: 422-469.

20 McClure HM, Ridley JH, Graham CE. Disseminated endometriosis in a Rhesus monkey (Macaca mulatta). Histogenesis and possible relationship to irradiation exposure $J$ Med Assoc Ga 1971; 60: 11-13.

21 Splitter GA, Kirk JH, Mac Kenzie WF, Rawlings CA Endometriosis in four irradiated rhesus monkeys. Vet Pathol 1972; 9: 249-262.

22 Wood DH, Yochmowitz MG, Salmon YL, Eason RL, Boster RA Proton irradiation and endometriosis. Aviat Space Environ Med 1983; 54: 718-724.

23 Fanton JW, Golden JG. Radiation-induced endometriosis in Macaca mulatta. Radiat Res 1991; 126: 141-146.

24 Marjoribanks J, Lethaby A, Farquhar C. Surgery versus medical therapy for heavy menstrual bleeding. Cochrane Database Syst Rev 2006: (2): CD003855.

25 Davis L, Kennedy S, Moore J, Prentice A. Modern combined oral contraceptives for pain associated with endometriosis. Cochrane Database Syst Rev 2007; (4): CD001019.

26 Collier J, Longmore M, Scally P. Oxford Handbook of Clinical Specialties (6th edn). Oxford, UK: Oxford University Press, 2003.

27 Frye CA. An overview of oral contraceptives: mechanism of action and clinical use. Neurology 2006; 66: S29-S36.

28 Ory HW. The noncontraceptive health benefits from oral contraceptive use. Fam Plann Perspect 1982; 14: 182-184.

29 Biering-Sorensen F, Bohr HH, Schaadt OP. Longitudinal study of bone mineral content in the lumbar spine, the forearm and the lower extremities after spinal cord injury. Eur J Clin Invest 1990; 20: 330-335.

30 Simoni RD, Hill RL, Vaughan M. Light is essential for bone deposition. Sunlight prevents rickets in rats. The work of A. M. Pappenheimer. J Biol Chem 2002; 277: e12-e18.

31 Bushinsky DA, Riordon DR, Chan JS, Krieger NS. Decreased potassium stimulates bone resorption. Am J Physiol Renal Physiol 1997; 272(6 Pt 2): F774-F780.

32 Drummer C, Friedel V, Börger A, Störmer I, Wolter S, Zittermann A, et al. Effects of elevated carbon dioxide environment on calcium metabolism in humans. Aviat Space Environ Med 1998; 69: 291-298.

33 Whedon GD, Lutwak L, Reid J, Rambaut P, Whittle M, Smith M, et al. Mineral and nitrogen metabolic studies on Skylab orbital space flights. Trans Assoc Am Physicians 1974; 87: 95-110.

34 Lang T. Cortical and trabecular bone mineral loss from the spine and hip in long-duration spaceflight. J Bone Miner Res 2004; 19: 1006-1012.

35 Recker RR, Davies KM, Hinders SM, Heaney RP, Stegman MR, Kimmel DB. Bone gain in young adult women. JAMA 1992; 268: 2403-2408.

36 DeCherney A. Bone-sparing properties of oral contraceptives. Am J Obstet Gynecol 1996; 174: 15-20.

37 Ravn P, Bidstrup M, Wasnich RD, Davis JW, McClung MR, Balske A, et al. Alendronate and estrogen-progestin in the longterm prevention of bone loss: four-year results from the early postmenopausal intervention cohort study: a randomized, controlled trial. Ann Intern Med 1999; 131: 935-942.

38 Dervay JP, Powell MR, Butler B, Fife CE. The effect of exercise and rest duration on the generation of venous gas bubbles at altitude. Aviat Space Environ Med 2002; 73: 22-27.

39 Waligora JM, Horrigan Jr DJ, Conkin J. The effect of extended $\mathrm{O}_{2}$ prebreathing on altitude decompression sickness and venous gas bubbles. Aviat Space Environ Med 1987; 58: A110-A112.

40 Krause KM, Pilmanis AA, Webb JT. The effect of menstrual day on decompression sickness (DCS) incidence in female research subjects. Aviat Space Environ Med 1998; 69: 199.

41 Rudge FW. Relationship of menstrual history to altitude chamber decompression sickness. Aviat Space Environ Med 1990; 61: 657-659.
42 Harm DL, Jennings RT, Meck JV, Powell MR, Putcha L, Sams $\mathrm{CP}$, et al. Invited review: gender issues related to spaceflight: a NASA perspective. J Appl Physiol 2001; 91: 2374-2383.

43 James TW, Nandini K, Andrew AP. Gender not a factor for altitude decompression sickness risk. Aviat Space Environ Med 2003; 74: 2-10.

44 Lee V, St Leger Dowse M, Edge C, Gunby A, Bryson P. Decompression sickness in women: a possible relationship with the menstrual cycle. Aviat Space Environ Med 2003; 74: 1177-1182.

45 Clemons M, Goss P. Estrogen and the risk of breast cancer. $N$ Engl J Med 2001; 344: 276-285.

46 Breast cancer and hormonal contraceptives: collaborative reanalysis of individual data on 53297 women with breast cancer and 100239 women without breast cancer from 54 epidemiological studies. Collaborative Group on Hormonal Factors in Breast Cancer. Lancet 1996; 347: 1713-1727.

47 Grunfeld EA, Price C, Goadsby PJ, Gresty MA. Motion sickness, migraine, and menstruation in mariners. Lancet 1998; 351: 1106

48 Reason J, Brand J. Motion Sickness. London, UK: Academic Press, 1975.

49 Golding JF, Kadzere P, Gresty MA. Motion sickness susceptibility fluctuates through the menstrual cycle. Aviat Space Environ Med 2005; 76: 970-973.

50 Bacic A, Rumboldt Z, Gluncic I, Buklijas J. The impact of the menstrual cycle and ondansetron on postoperative nausea and vomiting. Int J Clin Pharmacol Res 1998; 18: 153-158.

51 Clemes SA, Howarth PA. Changes in virtual simulator sickness susceptibility throughout the menstrual cycle. Proceedings of the 36th United Kingdom Group Conference on Human Response to Vibration, QinetiQ, Farnborough, UK, 12-14 September 2001. Farnborough, UK: QinetiQ, 2001; 190-202.

52 Kozarsky PE. Prevention of common travel ailments. Infect Dis Clin North Am 1998; 12: 305-324.

53 Grunfeld E, Gresty MA. Relationship between motion sickness, migraine and menstruation in crew members of a "round the world" yacht race. Brain Res Bull 1998; 47: 433-436.

54 Mercer A, Brown JD. Venous thromboembolism associated with air travel: a report of 33 patients. Aviat Space Environ Med 1998; 69: 154-157.

55 Chee YL, Watson HG. Air travel and thrombosis. Br J Haematol 2005; 130: 671-680.

56 Dinger JC, Heinemann LA, Kuhl-Habich D. The safety of a drospirenone-containing oral contraceptive: final results from the European Active Surveillance study on Oral Contraceptives based on 142,475 women-years of observation. Contraception 2007; 75: 344-354.

57 Farmer RD, Lawrenson RA, Thompson CR, Kennedy JG, Hambleton IR. Population-based study of risk of venous thromboembolism associated with various oral contraceptives. Lancet 1997; 349: 83-88.

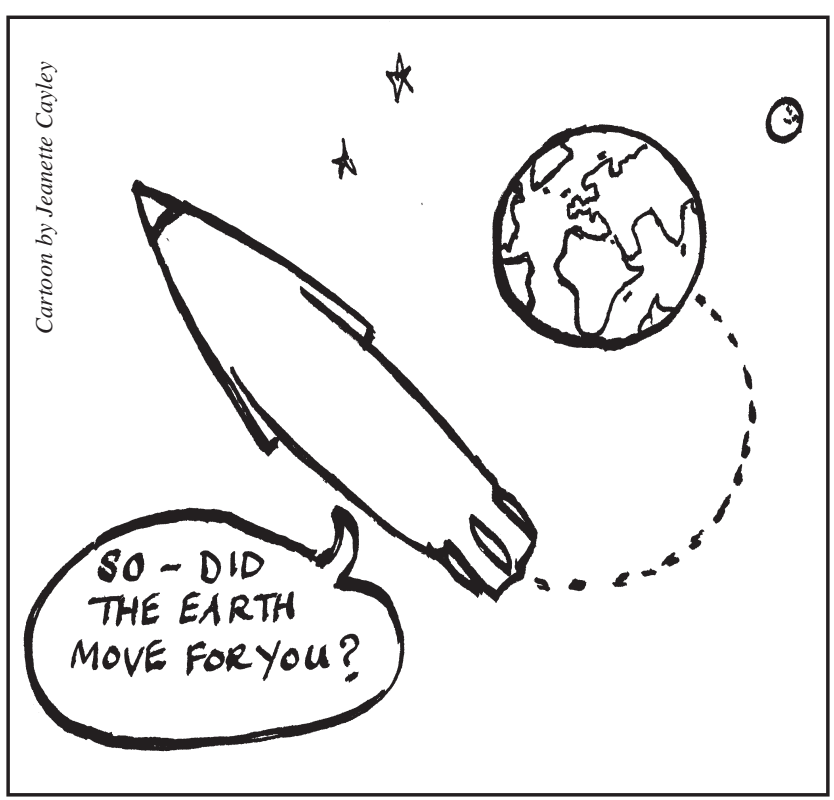

